\title{
Decision-Making Culture in Organizations
}

\author{
Hardi Mulyono, Nelvitia Purba , Arief Hadian, Abd. Rasyid Syamsuri, and Mhd. \\ Zulkifli Hasibuan
}

Universitas Muslim Nusantara Al Washliyah Medan, Indonesia

*Corresponding author email: nelvitiapurba@umnaw.ac.id

\begin{abstract}
This article discusses several definitions of decision making. Decision making is the process of choosing solutions from problem solving. The definition of decision making has been concluded that decision making is a process of determining an existing problem and then looking for some of the best alternatives available that can satisfy decision makers. The purpose of decision making is to make optimal results, make satisfactory results, make maximum results, make maximin results. The benefits of decision making that can be done are implementing managerial functions, establishing without decision making, evaluating managerial performance, assisting in making plans and policies, helping in choosing the best results from several alternatives by evaluating the strengths and weaknesses of decisions, helping the success of the organization's operations.
\end{abstract}

Keywords: Decision-Making, organization, culture

\section{INTRODUCTION}

Making decisions is a task of an organizational leader. Making subordinate decisions is done through a process by considering existing data or conditions which then considers the best conditions as an option to be determined as a decision choice. According to [1], a decision in management is a process of choosing between two or more alternatives to achieve one's desired goals. Managers at all levels of the organization make decisions to achieve a company goal and to survive in an organization [2]. Meanwhile, according to [3], decision making is a process that reduces uncertainty to a considerable degree. For most decisions, uncertainty is reduced rather than eliminated. Good decision making is an essential skill for career success in general and effective leadership in particular [4].

\section{LITERATURE REVIEW}

Decision making is very important thing in an organization, in profit organization or non-profit organization. According to [5], decision making is a process in determining the nature of the problem or opportunity that exists and choosing among the best alternatives available to solve problems or take advantage of opportunities. Decision making is a process of identifying and choosing between several alternative actions by agreeing - according to the demands of the situation [6].

Decision making is the process of choosing a solution from the best available alternative by making a decision [7]. Decision making is a process of identifying existing problems and opportunities and solving them or taking advantage of them [8]. Decision making is an intellectual process to choose the optimal and best option among the many alternative options available [9]. Decision making is a problem-solving process and ends when a satisfactory solution is reached, thus decision-making can be considered as an argumentative or emotional process that is carried out rationally based on implicit or explicit assumptions [10]. After describing some of the definitions of decision making by experts, we conclude that the definition of decision making is a process of determining an existing problem which looks for some of the best available alternatives that can satisfy the decision maker. 


\subsection{Purpose in Decision Making}

In making good decisions requires goals to be achieved. According to [3], the purpose of decision making are as follows.

1. Creating optimal results: this is a strategy in selecting options among several identified alternatives. The effectiveness of this strategy depends on how important the problem, the time limit available, the availability of available resources, whether there are other alternative costs, and the psychological state of the decision maker.

2. Creating satisfactory results: This strategy is carried out by considering the first satisfactory alternative rather than the best alternative. The word satisfying comes from two words, namely satisfying and sufficient. When these two words coexist, decision making can be considered as the best choice.

3. Making maximax results: This action is to do the maximizing of the maximum state. In this strategy, evaluation is carried out from several alternatives by selecting the maximum profit as the best option.

4. Making maximin results: This action is to do the maximization of the minimal state. Strategy is a pessimistic strategic action because it considers the worst possible outcome of all the alternatives and chooses the highest minimum. This type of strategy is used when failure is expensive and intolerable.

\subsection{Benefits of Decision Making}

In determining decision making, there are several benefits that can be obtained, according to [9], decision making is very important for an organization because it produces the best action with a cognitive process, the benefits of this decision are as follows.

1. Implementing managerial functions: many managerial functions such as planning, organizing, controlling, staffing, etc. It cannot be managed and directed properly.

2. Establish without decision-making, any function cannot be performed. It should be taken up by all staff.

3. Evaluating managerial performance. If it is true, it means that the workforce is working efficiently and correctly.

4. Assist in making plans and policies.

5. Assist in selecting the best outcome from several alternatives by evaluating their advantages and disadvantages.

6. Helping the success of the organization's operations.

\subsection{Factors that influence the decision making process}

In the decision-making process, the decision maker is influenced by several influencing factors, according to [11], these factors are as follows.

1. Rational factors: rational factors that are considered in making a decision are quantitative factors such as price, time, predictions, etc. In making decisions tend to consider these rational factors and often forget nonquantitative facts.

2. Psychological factors: Psychological factors are human psychological factors in participation in decision making. Psychological factors such as abilities, experiences, perceptions, values, goals, and roles are important factors in decision making.

3. Social factors: The agreement of other people, especially those who influence determinants, is an important issue. Taking these issues into account reduces the resistance of others to the decision.

4. Cultural factors: cultural factors such as the surrounding environment have several different layers consisting of regional culture, state culture, and natural culture. In addition, the organization also has an organizational culture which is a determining factor that must be considered. These cultures influence the decision making taken by individuals/organizations in the form of socially accepted values, trends, and general values.

\subsection{Techniques or Tools That Used In Making Good Decisions}

The decisions that are made can be good decisions and they can be bad decisions. In making a good decision result, according to [3], several techniques and tools that can be done are as follows.

1. Decision Tree: The decision tree is an excellent tool that can be used in making decisions. That the human eye is very easy to read an image, thus making a decision tree easier to read and understand. This evaluation is carried out at each decision node. Each decision tree node represents an alternative.

2. PMI technique: PMI which stands for 'Plus Minus Interesting'. It is an improvement on the pros and cons of techniques that have been used for centuries. PMI is used to evaluate the various options available by drawing a table with three columns to evaluate the plus or positive effects of alternatives in the first column, and negative effects in the second and third columns for other possible events, either positive or negative. These effects can be judged for better evaluation as 
some of them can be more subjective than others. This technique can be likened to a SWOT analysis.

3. Consultation: Consultation is the most commonly used evaluation technique. When consulting, the only thing to do is consult the right people and ask the right questions.

4. Experience: Experience is the best teacher. Evaluation in some ways can be based on past events and experiences. This is a good choice but a good analysis of the current and specific situation should be carried out, as certain changed conditions can produce different results.

5. Cost-Benefit Analysis: is a systematic way to calculate the benefits and costs of a project. It estimates an equivalent monetary value of a project. The downside of this technique is that it places so much value on money than any other risk or benefit that may be attached to the project.

6. Simulation: The purpose of simulation is beyond decision making because it can be used for performance optimization, safety engineering, testing and education. This action is a scientific action that is used to represent real world processes. Simulation actions are very important because existing past experiences and project knowledge are unknown. The downside of this technique is that it requires experts and people tend to rely heavily on the results because they appear real.

7. Linear Programming: Linear programming can be used in various fields of study. Usually applied to business and economics, but can also be used for some engineering problems. This mathematical method is used to determine the maximum profit or the lowest cost.

\subsection{Rational Models in Decision Making}

According to [12], rationality can be equated with scientific reasoning, empiricism and positivism and with the use of evidence decision criteria, logical argument and reasoning. Rational decisions based on rationality. The advantage of the classical model is that it shows a rational approach that can be applied to business to reach decisions in organizations [4]. According to [8], the stages of decision making are as follows.

1. Identify and Diagnose Problems. The first step in decision making is identifying the problem or opportunity. Opportunity is a condition where there is a desire to change the current condition for the better, while the problem is when the current condition is under expected condition. When the problem is identified, a search for the cause of the problem is carried out, by gathering information, exploring the causes of the problem, eliminating the causes of the problem, and focusing on the real causes of the problem.

2. Generating Alternative Solutions. The second step is to come up with a possible solution to the problem based on the perceived cause. There are several problems that can be resolved using programmed troubleshooting where there is a ready-made problem solution. However, there are conditions that are not programmed where decisions cannot be made because there is no problem solving procedure. In making decisions in this unprogrammed condition, it is necessary to produce creative alternative solutions.

3. Evaluating Alternatives. Stage three is to make a decision to examine alternative solutions using several decision criteria. The desired decision criteria must be in accordance with the existing objectives of the organization. After determining the criteria for decision making that is need to be done is to produce an alternative desired result

4. Choosing the Best Alternative. The next stage in decision making is to choose the best alternative by optimizing the best alternative from several predetermined criteria. In this case the decision maker will be satisfied after the alternative solution selection meets the minimum criteria or the decision maker has met an acceptable solution if the choice is not optimal.

5. Implementing Decisions. Implementing alternative solutions that have been decided and ensuring success is a continuation of the step of choosing the best alternative. Implementation requires the decision maker to put the solutions that have been decided into practice. The implementation or implementation of the best alternative option is a critical step because it requires the support and cooperation of all parties who will implement this choice.

6. Evaluating Results. The final step taken in decision making is evaluating the results of implementing the decision from the best alternative choice. The decision maker collects information and tries to learn from the decision from implementing alternative options that have the best achieved the desired goals. In evaluating the decision maker it is necessary to set reasonable goals and indicators of success in order to make good judgments about the effectiveness of the best alternative selection decision.

\section{RESULT AND DISCUSSION}

Research is a scientific activity by searching for various information. According to [13], scientific research is 
research conducted for the purpose of contributing to science by systematic and planned data collection, interpretation and evaluation. Research is a diligent systematic inquiry into nature and society to validate and refine existing knowledge and to generate new knowledge [14].

Research is the process of collecting, analyzing, and interpreting data to understand a phenomenon [15] Research is a structured process for collecting, analyzing, synthesizing, and interpreting (explaining or describing) data to answer theoretical questions that are not visible in the data [16].

According to [16], there are three ways that research can be used as material for the formulation of policy decisions based on the seven models proposed by Weiss, namely (1) using a rational approach, namely a model that is driven by knowledge and problem solving, this process is a rational policy where research will carried out if the decision maker needs it, (2) using a strategic approach, which considers research as ammunition to support a predetermined position or to postpone a decision, (3) uses an enlightenment approach or a diffusion approach, emphasizing that research and policy-making are carried out simultaneously with social processes other.

Meanwhile, according to [17], that there are three objectives as basis for research implementation design, namely (1) evaluation that focuses on utilization, which directs evaluation researchers to consider the research objectives intended by the intended users in the design and implementation of evaluation, evaluation that focuses on utilization emphasizes the use of research findings for the program, and highlights how stakeholder involvement in the evaluation process can support program and organizational design and development, (2) evaluation research includes a participatory evaluation model, in which stakeholders are involved in all aspects of evaluation research, from formulating questions to interpreting results, this model assumes that the knowledge people use to guide decision-making in organizations is socially constructed and that participation in all aspects of the evaluation process helps to develop a shared understanding of program goals, effects, and conditions for success, (3) theory driven evaluation emphasizes the importance of using a combination of social science and stakeholder theory in designing evaluation studies and using evaluation to support program planning, the main reasons behind this model are to be effective, interventions need to be based on theory and evidence from the relevant social science disciplines.

\section{CONCLUSION}

Decision making is a very important thing in an organization, in a profit organization or a non-profit organization. The decision maker is an action taken in solving problems and opportunities by taking some of the best alternatives by implementing the best alternative selection and finally evaluating its implementation. The purpose of decision making is to make optimal results, make satisfactory results, make maximax results, make maximin result.

The benefits of making decisions that can be done are implementing managerial functions, making decisions without making decisions, evaluating managerial performance, assisting in making plans and policies, assisting in choosing the best results from several alternatives by evaluating the strengths and weaknesses of decisions, helping the success of organizational operations. Factors that influence decision making are rational factors, psychological factors, social factors, and cultural factors.

Techniques used in decision making are using decision trees, using PMI techniques, using consultation, using experience, using cost and benefit analysis, using simulations, and using linnier programs. And the stages of decision making are identifying and diagnosing problems, producing alternative solutions, evaluating alternatives, choosing the best alternative, implementing decisions, evaluating results.

Research as an activity in the process of collecting, analyzing, and interpreting data to understand an existing phenomenon, thus research is a study material that can be considered in making decisions in organizations. So that decision makers in the organization can see judgment as a material that can be taken into consideration in making decisions.

\section{REFERENCES}

[1] O. H. Negulescu, Using a decision-making process model in strategic management. Review of General Management, 19 (1) (2014), 111-123.

[2] A. E. M. A. Shra'ah, The Impact of DecisionMaking Styles on Organizational Learning: An Empirical Study on the Public Manufacturing Companies in Jordan. International Journal of Business and Social Science, 6 (4) (2015), 55-62.

[3] M. T. Ahmed, H. Omotunde, Theories and Strategies of Good Decision Making. International Journal of Scientific \& Technology Research, 1 (10) (2012), 51-54. 
[4] B. Li, The Classical Model of Decision Making Has Been Accepted as not providing an Accurate Account of How People Typically Make Decisions. International Journal of Business and Management, 3 (6) (2008), 151-154.

[5] M. A. Hitt, J. S. Black, L. W. Porter, Management (third ed.). New Jersey: Prentice Hall, 2012.

[6] R. Kreitner, Management (eleventh ed.). Canada: Houghton Mifflin Harcourt, 2009.

[7] Chuck Williams, Management (fifth ed.). USA: South-Western Cengage Learning, 2009.

[8] L. R. Gomez-Mejia, D. B. Balkin, Management People/Performance/Change. New Jersey: Prentice Hall, 2012.

[9] D. Verma, Study and Analysis of Various Decision Making Models in an Organization. IOSR Journal of Business and Management (IOSR-JBM), 16 (2) (2014), 171-175.

[10] A. M. Shahsavarani, E. A. M. Abadi, The Bases, Principles, and Methods of Decision-Making: A Review of Literature. International Journal of Medical Reviews, 2 (1) (2015), 214-225.

[11] J. Nightingale, Think Smart-Act Smart: Avoiding The Business Mistakes That Even Intelligent People Make. John Wiley \& Sons, 2008.

[12] A. Huczynski, D. A. Buchanan, Organizational Behaviour. Prentice Hall, 2006.

[13] C. Ö. Çaparlar, A. Dönmez, What is Scientific Research and How Can it be Done?. Turkish journal of anaesthesiology and reanimation, 44 (4) (2016), 212218. doi: 10.5152/TJAR.2016.34711

[14] N. Naidoo, What is research? A conceptual understanding. African Journal of Emergency Medicine, 1 (1) (2011), 47-48.

[15] Carrie Williams, Research Methods. Journal of Business \& Economic Research, 5 (3) (2007), 65-72.

[16] J. Trostle, M. Bronfman, A. Langer, How do researchers influence decision-makers? Case studiesof Mexican policies. HEALTH POLICY AND PLANNING, $14 \quad$ (2) (1999), 103-114. doi: 10.1093/heapol/14.2.103

[17] B. J. Fishman, W. R. Penuel, A. R. Allen, B. H. Cheng, N. Sabelli, Design-Based Implementation Research: An Emerging Model for Transforming the
Relationship of Research and Practice. National Society for the Study of Education, 112 (2) (2013), 136-156. 